\title{
PESQUISAS DE ENFERMAGEM EM TERAPIA INTENSIVA: ANÁLISE DE DISSERTAÇÕES E TESES*
}

\author{
RESEARCH ON INTENSIVE CARE NURSING: \\ ANALYSIS OF THESES AND DISSERTATIONS
}

CORREA, A.K. Pesquisas de enfermagem em terapia intensiva: análise de dissertações e teses.

Rev.Esc.Enf.USP, v.34, n.3, p. 252-7, set. 2000.

\section{RESUMO}

A proposta deste trabalho é analisar as dissertações e teses nacionais realizadas na área de enfermagem em terapia intensiva. Para tal, foram consultados os resumos desses estudos publicados nos Catálogos do Centro de Estudos e Pesquisas em Enfermagem - volume 01 (1979) ao 15 (1997), buscando conhecer o número de trabalhos produzidos, local de defesa, temática e referencial teórico-metodológico utilizado.

PALAVRAS-CHAVE: Pesquisa. Unidades de Terapia Intensiva.

\section{ABSTRACT}

The proposal this study is to analyse national dissertations and theses on intensive care nursing The author consulted the Catalogues of the Centre of Studies and Research on Nursing - volume 1 (1979) to 15 (1997). The author analysed the abstracts and in order to search for: number of studies, place of presentation, theme and theoretical methodological reference.

KEYWORDS: Research. Intensive Care Units.

\section{INTRODUÇÃO}

No Brasil, nas décadas de 30, 40 e 50 eram escassas as pesquisas em enfermagem. O único periódico específico da área era a Revista Brasileira de Enfermagem (inicialmente denominada Anais de Enfermagem), criado pela ABEn em 1932. Os conhecimentos e informações contidos nos artigos dessa revista até a metade deste século não eram originários de pesquisa propriamente dita, mas sim de experiências pessoais, tradição e autoridade(7)

$\mathrm{O}$ conhecimento produzido na enfermagem foi aos poucos se diferenciando na busca de embasamento teórico-metodológico, o que foi impulsionado pela criação dos cursos de pósgraduação "sensu stricto", no Brasil, na década de 70, após a reforma universitária.(2)

A pesquisa passa então, nesse momento histórico, a ser produzida nos espaços acadêmicos das Universidades como, primordialmente, exigência da reforma universitária. Esse encaminhamento da investigação produz avanços, em termos quantitativos e qualitativos nas pesquisas de enfermagem mas, ao mesmo, suscita outros questionamentos que permanecem até a década atual. Dentre esses podemos mencionar: as pesquisas realizadas vêm acompanhando e respondendo às necessidades advindas da prática de enfermagem e, mais que isso, às necessidades de saúde e qualidade de vida da população? Como articular os serviços e a universidade na produção de conhecimentos? Como os pesquisadores têm se posicionado no contexto sócio-político-econômico e no setor saúde?

* Artigo adaptado de trabalho apresentado à disciplina de pós-graduação "Análise Crítica da Evolução da Assistência de Enfermagem"do Programa Doutorado Interunidades, Escola de Enfermagem de Ribeirão Preto, Universidade de São Paulo. Apresentado, na forma de pôster, na $6^{a}$ Reunião Especial da SBPC, realizada em Maringá (PR), em outubro de 1998.

* Enfermeira, Professora Assistente do Departamento de Enfermagem Geral e Especializada da Escola de Enfermagem de Ribeirão Preto, Universidade de São Paulo. E-MAIL: adricor@glete.eerp.usp.br 
Diante desses questionamentos compreendo que a pesquisa deva ser constantemente analisada, evitando que ela própria se torne um pensar mecanizado, reprodutor de práticas já consagradas.

Nesse sentido, a produção de conhecimentos em terapia intensiva é uma temática que me inquieta, tendo em vista a minha experiência profissional prévia nessa área de assistência e meu interesse ainda presente em analisar, de modo crítico, a prática de enfermagem aí realizada.

Nesse contexto, a proposta deste trabalho é analisar as dissertações e teses de enfermagem realizadas na área de terapia intensiva, no Brasil, buscando conhecer o número de trabalhos produzidos, local de defesa, temáticas enfocadas e referenciais teórico-metodológicos que vêm sendo utilizados.

\section{METODOLOGIA}

Para realizar este trabalho, foram consultados os Catálogos do Centro de Estudos e Pesquisas em Enfermagem (CEPEN) - volume 1 (1979) ao volume 15 (1997). Nesses catálogos constam trabalhos defendidos até 1996 (ver anexo 01)

Foi realizada a leitura dos resumos de dissertações e teses que se referiam à terapia intensiva. $\mathrm{Na}$ maioria das vezes, o título do trabalho já explicita que se trata de um estudo nessa área, em alguns casos, tal explicitação só se dá no decorrer do resumo.

Através de leitura cuidadosa de cada resumo, foram buscados os seguintes aspectos: número de trabalhos desenvolvidos em cada década, local de defesa, temática estudada e referencial teóricometodológico.

\section{A PRODUÇÃo CIENTÍFICA DE ENFERMAGEM EM TERAPIA INTENSIVA}

Foram encontrados nos Catálogos do CEPEn quarenta e seis trabalhos (46) relativos à terapia intensiva, sendo quarenta e duas (42) dissertações de mestrado e quatro (04) teses de doutorado.

$\mathrm{Na}$ década de 70 , não foi produzida nenhuma dissertação ou tese sobre terapia intensiva. Acredito que a ausência desses estudos, nesse período, se relaciona, principalmente, com o advento da terapia intensiva no Brasil, somente a partir dessa década.
Esse era o período do "milagre econômico", no qual a politica caracterizava-se, essencialmente, pela busca de modernização, também no setor saúde. Assim, ocorriam a absorção de avanços tecnológicos, recursos farmacêuticos e de equipamentos, bem como o desenvolvimento de métodos de diagnóstico e terapia (3). Ou seja, este contexto político encaminha as ações de saúde para o predomínio da assistência médica curativa individual, emergindo com intensidade as especialidades no complexo médico hospitalar.

Esse era um momento, portanto, no qual a preocupação dos enfermeiros centrava-se, provavelmente, na organização da prática nesse novo campo de trabalho, não estando ainda voltada para o aprofundamento de conhecimentos nessa área.

Cabe ainda considerar que os cursos de pósgraduação começaram a se estruturar no país somente a partir da década de 70 , como já comentado, e foram esses que muito impulsionaram a produção científica da enfermagem brasileira.

Nas décadas de 80 e 90, os enfermeiros que de algum modo se relacionavam com a prática em terapia intensiva já buscavam aperfeiçoar sua prática, prática essa que se mantém coerente ao modelo de saúde vigente. Assim, na década de 80 foram produzidas quatorze dissertações de mestrado (14) e uma tese de doutorado (01). Na década de 90 (lembrando que a análise foi realizada até 1996) foram realizadas vinte e oito dissertações de mestrado (28) e três teses de doutorado (03). Desse modo, a produção cientifica de enfermagem em terapia intensiva, através de dissertações e teses, tem aumentado.

A maioria das dissertações de mestrado foram produzidas na Escola de Enfermagem Ana Néry (RJ) e na Escola de Enfermagem da Universidade de São Paulo (SP). Os demais trabalhos se distribuem em diferentes locais como: Escola de Enfermagem de Ribeirão Preto da Universidade de São Paulo, Escola de Enfermagem da Universidade Federal de Santa Catarina, Universidade Federal da Bahia, Escola de Enfermagem da Universidade do Rio de Janeiro, Escola Paulista de Medicina, Instituto de Psicologia da Universidade de Brasília, Instituto de Filosofia e Ciências Sociais da Universidade Federal do Rio de Janeiro e Centro de Ciências da Saúde da Universidade Federal da Paraíba.

A tabela a seguir ilustra essa distribuição em cada década. 
Tabela 1- Dissertações de Enfermagem em Terapia Intensiva Segundo

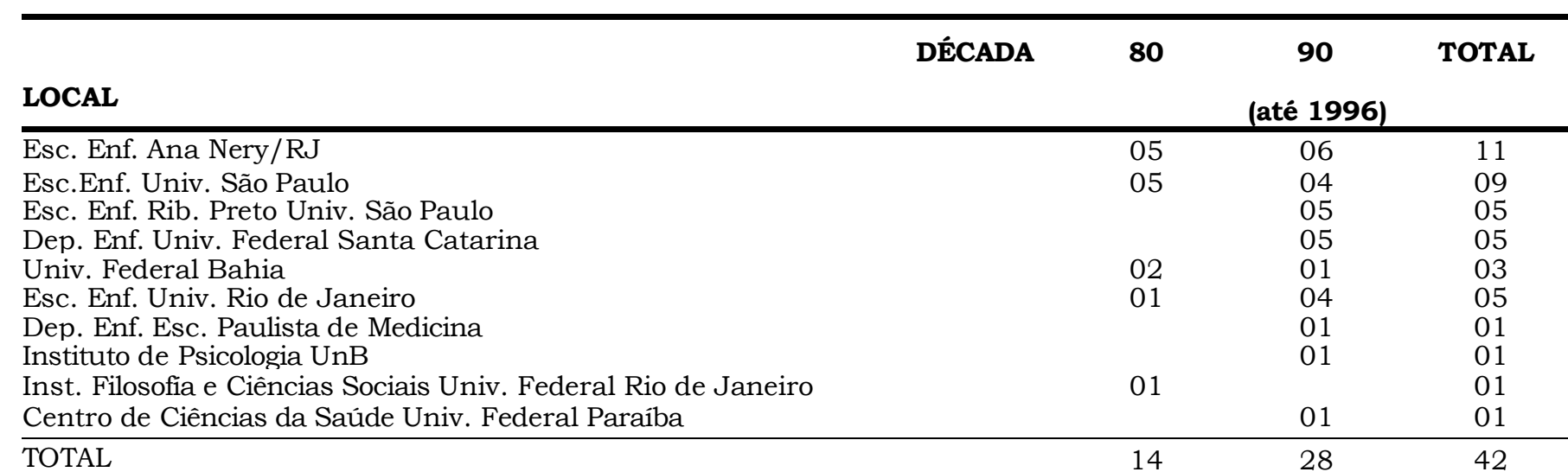

Em relação às teses de doutorado, todas foram produzidas na Escola de Enfermagem da Universidade de São Paulo, sendo uma defendida na década de 80 e as demais na década de 90. Levando-se em conta tais dados, essa escola produziu, segundo consta nos Catálogos do CEPEn, 13 trabalhos em terapia intensiva (dissertações e teses).

Torna-se importante questionar porquê a maioria dos trabalhos são provenientes das Escolas de Enfermagem da Universidade de São Paulo e Ana Néry.

Não se pode deixar de considerar, inicialmente, que nesses locais se desenvolveram os primeiros cursos de mestrado na área de enfermagem do país (Ana Néry em 1972; Esc. Enf. USP em 1973)(1) tendo adquirido tradição em pesquisa precocemente. Além disso, são grandes centros que polarizam alunos de todo o país.

Nesse mesmo raciocínio é interessante que, apesar da Escola de Enfermagem de Ribeirão Preto - USP também ser um grande centro de pesquisa em enfermagem, foram aí desenvolvidos apenas cinco (05) trabalhos em terapia intensiva (dissertações de mestrado).

Nesse sentido, a análise do predomínio de alguns locais na produção de trabalhos em terapia intensiva, envolve considerar aspectos como: temáticas de pesquisa que vêm se delineando em cada escola e o envolvimento de docentes-pesquisadores com essa prática, o que se relaciona com a condução do currículo de graduação e com os cursos de especialização. Nesse momento, não disponho de informações mais consistentes que me permitam aprofundar tal análise.

Deve ser ressaltado, ainda, que a região sudeste é pólo de atendimento especializado em saúde. A terapia intensiva é, predominantemente, uma realidade da região sudeste, do estado de São Paulo e do municipio de São Paulo."'

A partir da leitura dos resumos, os trabalhos se configuraram em nove (09) temáticas gerais. Cabe ressaltar que essas temáticas emergiram dos próprios trabalhos, não sendo formuladas previamente. São elas:
- A prática do enfermeiro (13)*

- A familia do paciente (09)

- Os problemas dos pacientes internados (06)

- A sistematização da assistência de enfermagem (05)

- A avaliação de técnicas e cuidados de enfermagem (04)

- A percepção dos pacientes (04)

- A comunicação com os pacientes (02)

- O trabalho da equipe de enfermagem (01)

- Infeocãa hospitalar (01)

- O processo de socialização de novos membros (01)

Em estudo realizado por KOIZUMI et al.(6), no qual foram analisados artigos de enfermagem em terapia intensiva nas Revistas Brasileira de Enfermagem, Nursing Research e Heart \& Lung, de 1975 a 1984, as temáticas mais estudadas foram a avaliação de procedimentos de enfermagem ou terapêuticos e de parâmetros fisiológicos.

PADILHA et al(8), analisaram as publicações de onze (11) periódicos nacionais de enfermagem, no período de 1985 a 1994 e constataram que $2,7 \%$ eram referentes à enfermagem em terapia intensiva, sendo os assuntos mais enfocados: necessidades e problemas do paciente, metodologia de assistência e processo ensino-aprendizagem.

Neste estudo é a temática "a prática do enfermeiro" que se mostra como objeto de pesquisa que vem recebendo mais atenção nas dissertações e teses. Nessa temática são abordadas questões que se voltam para a prática cotidiana, numa busca de apreensão do trabalho do enfermeiro: as suas competências, a prática administrativa, as ocorrências iatrogênicas, as relações de poder, disputas e conflitos, o estresse, a emoção, enfim, os sentimentos e percepções em relação ao seu trabalho. 
As análises do trabalho do enfermeiro, de modo geral, restringem-se à internalidade de sua prática, entretanto, alguns estudos apontam para outras direções, enfocando algumas questões sociais e filosóficas. Sem dúvida, as temáticas estudadas são pertinentes e necessárias à compreensão do trabalho em terapia intensiva. Todavia, outros questionamentos se impõem nesse momento. Por exemplo, como compreender o trabalho em terapia intensiva, em relação às políticas e práticas de saúde atuais? Quais as concepções de saúde-doença e qualidade de vida veiculadas por essa prática?

A família do paciente também é uma temática que vem recebendo atenção por parte dos pesquisadores, sendo bastante enfocadas questões concernentes à internação de recém-nascidos e crianças. Além disso, são também focalizadas a relação estabelecida entre equipe de enfermagem

e família e as orientações que essa recebe.

Em relação à temática "os problemas dos pacientes", os estudos se voltam, prioritariamente à esfera física. Entretanto, o olhar dos pesquisadores também se dirigem para "a comunicação com os pacientes", bem como para a compreensão das suas próprias "percepções", tendo em vista as suas experiências enquanto paciente de terapia intensiva.

Do que foi exposto até então, compreendo que os enfermeiros que pesquisam em terapia intensiva vêm se despertando para outras dimensões, além da biológica, que configuram o fazer cotidiano do enfermeiro e o cuidar do paciente.

A sistematização da assistência de enfermagem é tema de algumas pesquisas. Essa tentativa de padronizar modelos, fundamentados em teorias de enfermagem, para o cuidar em terapia intensiva, muitas vezes, pode ser viável, considerando as especificidades desse setor no que diz respeito ao número de pacientes internados, número de profissionais e atividades realizadas. Planejar a assistência se faz necessário, no entanto, é preciso ir além, questionando as concepções de homem, saúde, doença e enfermagem incorporadas por tais modelos e as repercussões da sua utilização na qualidade da assistência prestada.

A "avaliação de técnicas e cuidados de enfermagem" é sempre importante em terapia intensiva, considerando a gravidade dos pacientes e a realização permanente de meios diagnósticos, de vigilância e terapêuticos invasivos. Penso que é ainda insuficiente os estudos dessa natureza, sendo preciso investimento na busca de conhecimentos que proporcionem fundamentação clínica consistente ao enfermeiro.

As temáticas por si mesmas revelam algumas áreas de interesse para estudo. Para apreender como o enfermeiro dirige seu olhar para as mesmas, torna-se necessário conhecer quais referenciais metodológicos vêm subsidiando as pesquisas. Esses referenciais permitem que os objetos de pesquisa sejam compreendidos sob perspectivas distintas, conforme o sentido que imprimem à investigação.

Em relação aos referenciais teóricometodológicos das pesquisas de enfermagem em terapia intensiva realizadas na forma de dissertações e teses, merecem destaque os seguintes aspectos:

- Dezoito (18) trabalhos utilizaram o referencial metodológico positivista;

- A fenomenologia foi mencionada como referencial metodológico de quatro (04) trabalhos;

- Três (03) estudos utilizaram o referencial metodológico das representações sociais;

- Um estudo (01) fundamentou-se no interacionismo simbólico e na etnografia;

- Outros cinco estudos (05) mencionaram, respectivamente, como referenciais teóricos: Foucault, Watson e Cassirrer, Morin e Capra, os príncipios da ergonomia, Agnes Heller.

- Dois (02) resumos apenas mencionaram que o estudo é de natureza qualitativa e outros dois (02), quanti-qualitativa;

- Onze (11) trabalhos não deixaram claro, em seu resumo, o referencial teórico-metodológico utilizado. Dentre esses estudos e aqueles denominados de natureza qualitativa, muitas vezes, são enfatizadas apenas técnicas de coleta de dados, como entrevistas e observações.

ROCHA; SILVA(9), analisando as dissertações e teses de enfermagem, a partir dos seus resumos nos Catálogos do CEPEn (de 1979 a 1984) apreendem a influência marcante do positivismo. Enfocam a tendência, na época, de propostas alternativas, como o materialismo histórico e dialético e a fenomenologia.

De certo modo, acredito que os estudos de terapia intensiva acompanham o movimento de produção científica na enfermagem, em relação aos referenciais teórico-metodológicos. Ou seja, no início dos anos 80, predominam estudos positivistas, cabendo salientar que treze (13) dos dezoito (18) trabalhos positivistas foram desenvolvidos na década de 80.

Nos primeiros cursos de mestrado em Enfermagem no Brasil predominava o modelo biomédico de pesquisa que já era adotado em programas similares em outros países, particularmente, nos Estados Unidos. Desse modo, no início, foi dada grande ênfase aos métodos quantitativos na produção das dissertações. (5) 
Posteriormente, outros paradigmas vão sendo incorporados às pesquisas, enquanto possibilidades outras de abordar os objetos de pesquisa.

Cabe considerar que a escolha do referencial teórico-metodológico relaciona-se ao problema a ser investigado e à medida que os enfermeiros pesquisadores da área de terapia intensiva começam a voltar-se para problemas que não são suficientemente aclarados com o modelo positivista, outras opções metodológicas vão sendo utilizadas, ampliando as perspectivas de compreensão. Ao mesmo tempo é importante ressaltar que a escolha desses referenciais relaciona-se à "visão de mundo" do pesquisador.

Penso que compete aos pesquisadores evitar que a incorporação de "novas" abordagens teóricometodológicas se restrinja ao plano técnico, ou seja aos procedimentos das pesquisas, com incipiente aprofundamento das bases teórico-filosóficas dos referenciais metodológicos. Como explicitado anteriormente, é significativo o número de resumos que somente apontam as técnicas de coleta de dados.

Cabe ainda considerar que os estudos realizados, na sua maioria, descrevem e analisam situações e alguns propõem modelos - comunicação terapêutica, sistematização da assistência, orientação pré-operatória - como talvez, tentativas de se adequar a prática a ideais pré-estabelecidos. Entretanto apenas a leitura dos resumos efetuada neste artigo não é suficiente para subsidiar uma discussão acerca da repercussão desses estudos na prática cotidiana

Nesse contexto, emerge a problemática da utilização do conhecimento produzido no meio acadêmico, na prática dos serviços. Este estudo não possibilitou a análise da autoria dos trabalhos, mas, como comentado por KOIZUMI(5), o produtor de pesquisa, muitas vezes é o aluno dos cursos de mestrado e doutorado, o qual, por sua vez, é um docente de uma universidade.

Produção de conhecimento e assistência, academia e serviço estão relacionados, porém são partes distintas de um todo. São estruturalmente diferentes e convivem cercados de interesses diversos. Buscar formas de articulação entre essas partes é um grande desafio que se impõe aqueles que se dedicam às pesquisas, em qualquer campo de atuação na enfermagem brasileira.

\section{CONSIDERAÇÕES FINAIS}

A análise da produção científica da enfermagem em terapia intensiva aponta caminhos já percorridos e suscita reflexões. Os resumos analisados apontam a preocupação dos pesquisadores com a prática do enfermeiro e o cuidar em terapia intensiva, para além das questões técnico-biológicas e a busca de abordagens metodológicas que permitam o estudo dos objetos de pesquisa sob distintas perspectivas.

A prática em terapia intensiva oferece um leque de temáticas possiveis de serem pesquisadas pelos enfermeiros, na busca de qualificar o fazer cotidiano

Penso que a busca de compreensão e qualificação dessa prática necessita ainda de estudos, dentre outros, que aprofundem a abordagem da terapia intensiva inserida no contexto de saúde atual, das concepções de saúdedoença aí predominantes, da qualidade do cuidado de enfermagem, bem como estudos que ampliem o saber clínico do enfermeiro, subsidiando um fazer crítico e competente.

\section{REFERÊNCIAS BIBLIOGRÁFICAS}

1.ALMEIDA, M.C.P. et al. A produção de conhecimento na pósgraduação em enfermagem no Brasil. In: CONGRESSO BRASILEIRO DE ENFERMAGEM, 33. Manaus, 1981. Anais. Manaus, Associação Brasileira de Enfermagem, 1981, p.11926.

2.ALMEIDA, M.C.P. A pós-graduação em enfermagem no Brasil: situação atual. Rev. Lat. Am. Enf., v.1, n.1, p.43-50, 1993.

3.IDE, C.A.C. Prática de enfermagem em UTI e contexto de saúde. Rev Esc. Enf. USP, v.23, n.1, p.91-8, 1989.

4.KIMURA, M. ; MIYADAHIRA, A.M.K. Aspectos da assistência hospitalar no município de São Paulo: a situação da assistência intensiva. Rev. Esc. Enf. USP, v.25, n.1, p.61-72, 1991.

5.KOIZUMI, M. S. Perfil da pesquisa de enfermagem em terapia intensiva no Brasil. Florianópolis, 1996. Conferência realizada na V Jornada Sul Brasileira de Terapia Intensiva, outubro, 1996.

6.KOIZUMI, M.S. et al. Análise retrospectiva das pesquisas de enfermagem em terapia intensiva: 1975-1984. Rev. Esc. Enf. USP, v.20, n, 1, p. 5-17, 1986.

7.MENDES, I.A. C. Pesquisa em enfermagem: impacto na prática. São Paulo, EDUSP, 1991.

8.PADILHA, K.G. et al. Produção científica de terapia intensiva no Brasil: análise retrospectiva de 1985 a 1994. In: ENCONTRO DE ENFERMAGEM E TECNOLOGIA, 5. São Paulo, 1996. Programa, São Paulo, 1996, p.384-88.

9.ROCHA, S.M.M.; SILVA,G.B. Linhas fillosóficas e ideológicas na pesquisa em enfermagem no Brasil. Rev. Bras.Enf., v.40,n.4, p.214-20, 1987. 


\section{ANEXO 01}

Catálogos do Centro de Estudos e Pesquisas em Enfermagem (CEPEn)

volume I - 1979

volume II - 1980

volume III - 1983

volume IV - 1984

volume V - 1985

volume VI - 1986

volume VII - 1986 (remissivo de 79 a 86)

volume VIII - 1989

volume IX - 1991

volume X - 1992

volumeXI - 1993

volume XII - 1994

volume XIII - 1995

volume XIV - 1996

volume XV - 1997 